\title{
PROCESO TRANSFORMACIONAL DEL PERSONAJE DEL AMO EN CONCIERTO BARROCO
}

\author{
POR \\ RAQUEL AGUILU DE MURPIIY \\ Marquette University
}

La novela Concierto barroco ${ }^{1}$ fue una de las últimas producciones literarias de Alejo Carpentier. Dado que hasta el presente, la crítica se ha ocupado de estudiar dos elementos en particular: la música y la presencia de América en la obra según queda plasmada en el espacio narrativo ${ }^{2}$, aún resta por hacerse un estudio sobre el proceso transformacional de la identidad del personaje del Amo en la novela. De estudiarse la función narrativa del Amo, sus acciones físicas, mentales, actitudes sicológicas e interacción nos remiten al núcleo básico del discurso narrativo; tal es, el motivo del viaje como búsqueda y definición de la identidad. En el caso del Amo esa búsqueda produce una transformación en el personaje al retroceder históricamente y auto-analizar su doblez cultural americana/europea. El viaje viene a representar la búsqueda de un modelo mítico europeo, un paraíso imaginado por el personaje en busca de sus propias raíces y por lo tanto, de su auto-definición e identidad.

Primero que nada hay que especificar que el viaje, como eje narrativo articulado por los cambios de espacio e interacciones sociales del Amo, producirá dualidades sicológicas y mentales en la concepción de los espacios y objetos del personaje, como se puede observar en el siguiente pasaje:

—“Aquí lo que se queda - decía el Amo-. Y acá lo que se va". En lo que se iba, también alguna vajilla menor, un juego de copas y, desde luego, la bacinilla del ojo de plata- (pp. 9-10).

La preparación de su viaje a Europa nos presenta la "realidad" espacial del protagonista dividida entre acáallá, lo que se va y lo que se queda, Américal Europa. Pero la ambigüedad de la auto-definición entre ser americano o

\footnotetext{
${ }^{1}$ Alejo Carpentier, Concierlo barroco (Madrid: Siglo Veintiuno, 1974) Las restantes citas provienen de esta edición.

${ }^{2}$ Hortensia Morell-Chardón, "Funcionalidad del motivo del "moro" en la composición "Concierto Barroco", Sin nombre, July-Sept. 12-2 (1981): 60-66; José Lu is Vega, "Tiempo, ritmo e historia en "Concierto Barroco", Sin nombre, July-Sept. 12-2, (1981): 67-80.
} 
europeo se fija y se emblematiza en la naturaleza misma proyectada en la pintura híbrida colgada en su casa:

Pero el cuadro de las grandezas estaba all ... donde historiábase ... el máximo acontecimiento de la historia del país. Alli, un Montezuma entre romano y azteca, algo César tocado con plumas de quetzal ... (p 11).

Este cuadro contiene la problemática histórica central de la novela, y por ende del personaje que, en este momento, está en la imposibilidad de distinguir los elementos de la historia mexicana de los elementos que caracterizan la historia imperialista europea, es decir, no hay una versión auténtica de la historia latinoamericana; sólo el discurso europeo oficial. Esta problemática se plasma aquí a dos nivèles: al nivel del personaje y al nivel del cuadro. De ahí su crisis, su búsqueda de identidad, su identificación de ser americano a lo largo del texto/del viaje. El cuadro es el elemento ambivalente ${ }^{3}$ de la narración que desdobla la función dual del personaje: "un Montezuma entre romano y azteca ..." (p. 11), ya que da énfasis al enfrentamiento de los dos mundos de Cortés y Montezuma, y a la vez ese pasado histórico problematiza la realidad inmediata del Amo como americano.

Terminados los preparativos del viaje, el Amo sale en su jornada hacia Europa, siendo Cuba su primer destino. Cuba representa en el texto una función histórica espacial, ya que, si la consideramos desde el punto de vista de la conquista de América, la isla (en la realidad histórica) representa el punto de partida de Hernán Cortés en su proyecto de conquistar a México. Para el Amo, en cambio, Cuba será el punto de salida en su viaje inverso al de Cortés y en sus vuelta al origen europeo: la ubicación en el comienzo del espacio que llevó a los agentes históricos a la conquista y colonización de América.

La llegada del Amo a Cuba va a causar en el amo el primer choque desilusionador con la historia oficial y canónica, al encontrar a la isla destruida y azotada por la peste y percibir la diferencia en la planificación urbana y percatarse del estilo arquitectónico estructural de los domicilios:

Aquí en cambio, las calles eran angostas, de las casas bajas, cuyas ventanas, en vez de tener cancelas de buen herraje, se abrían de varillas mal pintadas de blanco, bajo tejados que, en Coyoacán apenas si hubiesen servido para cobijar gallineros o porquerizas ... (p. 18).

Este enfrentamiento con esa realidad distinta a la ya conocida por el Amo le hacen comparar su tierra natal con la cubana, sin encontrar en ella nada que le cause agrado. Hay una desvalorización espacial, pues lo que ve allí sólo sirve, en su propio país, para cobijar animales. Una vez en Cuba, Francisquillo, el

${ }^{3}$ Julia Kristeva, El texto en la novela, trad. Jordi Llovet (Barcelona: Editorial Lumen, 1974) 124. 
criado, muere a causa de la peste, $y$, esto crea la necesidad de buscar a otro acólito que lo sustituya, pues para ser amo se necesita un criado, ya que dentro de esta sociedad burguesa europea/americana "un amo sin criado" no es "un amo de verdad" (p. 19). Filomeno viene a ocupar la posición de Francisquillo. Este nuevo criado negro consolidará la figura del Amo dentro de la sociedad aristocrática europea renacentista, pues "es moda de ricos señores tener pajes negros" (p. 20). La figura del criado negro le permite al Amo mantener su posición social dentro del modelo jerárquico y racista socio-cultural europeo/ americano.

Una vez empleado un nuevo criado, el Amo proseguirá su viaje. Llega a España donde le cuentan historias maravillosas de sus antepasados europeos. El esperaba encontrar una tierra llena de maravillas como las de México pero se desilusiona ante la imagen de la España de sus antepasados heroicos. Esto conduce al personaje a un segundo confrontamiento (como el de Cuba) con la realidad y el discurso oficial mítico de la historia pasada, lo cual genera una nueva desilusión:

Nieto de gente nacida en algún lugar situado entre Colmenar de Oreja y Villa Manrique del Tajo y que por lo mismo le habían contado maravillas de los lugares dejados atrás, imaginábase el Amo que Madrid era otra cosa. Triste, deslucida y pobre le parecia esa ciudad, después de haber crecido entre plazas y tezontles de México (p. 27).

La tierra extranjera se va convirtiendo en un lugar ambivalente y ambiguo. Para el Amo lo autóctono y lo extranjero están en oposición. Hay una valoración de ese otro espacio, España, que se acentúa más claramente cuando el Amo piensa en su propio espacio de origen, México. Este primer contacto con lo español - lo añorado y hasta imitado por el Amo en México - se va transformando, y adquiere una significación negativa. Esa transformación espacial también va creando una ambivalencia en el personaje. Se perfila una oscilación entre el Amo en su des-mitificación del modelo-espacio cultural mítico europeo y la aceptación del mexicano "con nostalgia" por lo mexicano, lo dejado atrás, su experiencia histórica como americano. El Amo compara, entonces, lo español con la tierra natal a través de la descripción de los diversos espacios ambientales y platos españoles:

Fuera de la Plaza Mayor, todo era, aquí, angosto, mugriento y esmirriado, cuando se pensaba en la anchura y el adorno de las calles de allá .... De cocina no podía hablarse: ante las albóndigas presentes, la monotonía de las merluzas, evocaba el mexicano la sutileza de los peces guachinangos y las pompas del guajilote vestido de salsas obscuras con aroma de chocolate y calores de mil pimientas ... (p. 28). 
Esa oposición empirica entre lo de aquí y lo de allá se va acentuando a medida que el Amo comienza a aburrirse de su estancia en Madrid: "Aquí las ferias no tenían el color ni la animación de las de Coyoacán .... Pasaban los días, y el Amo, con tanto dinero como traía, empezaba a aburrirse tremendamente" (p. 29). Decide, por lo tanto, acortar su estadía en España, -acción que instituye el rechazo - para llegar a Italia, y es aquí donde surge el primer conflicto entre amo y criado:

... si Filomeno se relamía de gusto recordando su primer festín de carne blanca, el Amo, seguido por una chusma de mendigos apenas aparecía en las calles donde ya era conocida la pinta de su jarano con recamados de plata, no cesaba en sus lamentos contra la ruindad de esta villa harto alabada ... (p. 29).

La inconformidad e inadaptabilidad del Amo en España lo hacen trasladarse a Venecia que en consecuencia establece una tercera transposición y dislocación espacial en el texto: Venecia. En este espacio ha comenzado el carnaval, "el gran carnaval de la Epifanía". El carnaval es un espectáculo simbólico y atemporal en donde reina la anomalía, multiplicándose las confusiones, la excentricidad y la ambivalencia ${ }^{5}$. Es por tanto que el carnaval constituye en la novela otro mediador transformacional en el personaje del Amo, ya que al utilizar la máscara pierde su identidad inicial de europeo para sumergirse en la de su pasado histórico-mexicano:

$Y$ todo el mundo, entonces cambió de cara. Antifaces de albayalde, todos iguales, petrificaron los rostros de los hombres de condición, entre el charol de los sombreros y el cuello de tabardo; antifaces de terciopelo obscuro ocultaron el semblante sólo vivo en labios y dientes, de las embozadas de pie fíno (p. 34).

\footnotetext{
${ }^{4}$ A Filomeno le resulta muy agradable la permanencia en España, porque para él es una apertura a lo nuevo, lo antes desconocido. Mientras que, para el Amo, la estancia en España resultará, por el contrario, insoportable y negativa.

${ }_{5}$ Bakhtine, Mikhail, Rabelais And His World, translated by Helen Iswolsky (Indiana University Press, 1984) 7-8. Complementamos esta interpretación del carnaval con la definición de Bakhtin. Al respecto Bakhtin dice lo siguiente:

Carnival is not a spectacle seen by the people; they live in it, and everyone participates because its very idea embraces all the people. While carnival lasts, there is no other life outside it. During carnival time life is subject only to its laws, that is, the laws of its own freedom. It has a universal spiril; it is a special condition of the entire world, of the world's revival and renewal in which all take part. Such is the essence of carnival, vividly felt by all participants. It was most clearly expressed and experienced in the Roman Saturnalias, perceived as a true and full, though temporary, return of Saturn's golden age upon earth. The tradition of Saturnalias remained unbroken and alive in the medieval carnival, which expressed this universal renewal and was vividly felt as an escape from usual official way of life... Thus, carnival is the people's second life, organized on the basis of laugther. It is a festive life. Festivity quality of all comic rituals and spectacles of the Middle Agges.
} 
El propósito de la máscara es servir de mecanismo de separación del personaje con respecto al mundo, pasado mexicano y el europeo ${ }^{6}$. Así, la máscara se convierte en un instrumento de oposición entre el pasado y el ambiente local que le rodea, dado que el Amo participa en el carnaval asumiendo la personalidad de Montezuma. La máscara y la vestimenta pasan a ser agentes mediadores de la transformación del personaje en la búsqueda de su identidad americana. Al llegar el Amo vestido de Montezuma al "Botteghe di Caffe", el fraile Pelirrojo le pregunta, "¿Inca?" y aquél responde, "Mexicano". Volvemos, pues, a perfilar la ambivalencia del personaje al contestar mexicano, pues este nombre encarna tanto lo indio como lo español, sin ser el uno ni el otro, pero si, todos a la vez -indio y europeo-.

Luego el Amo cuenta la historia de la conquista de México, "historia" que interesará al fraile Pelirrojo como tema para una ópera. De aquí en adelante el Amo asume la personalidad integral de Montezuma por medio de la máscara, es decir, se enmascara para asumir la personalidad que le identifica con sus pasado histórico autóctono. Sin embargo, la imagen del Amo no desaparece por completo; la máscara viene a consolidar en una unidad integradora tanto la figura del Amo como la de Montezuma, lo americano y lo europeo que desdobla al mestizo futuro. Así también el tiempo se transforma por mediación de la máscara. Pasado y presente se unifican en el personaje por medio de la máscara posibilitando un acercamiento y a la vez distanciamiento histórico-temporal, necesarios para "alejarse de las cosas ... para ver las cosas más de cerca", y de esa forma volver a revivir "épocas por siempre idas" (p. 76), como puede observarse en la siguiente cita:

-"Mexicano" - respondio el Amo, largándose a contar una larga historia que el fraile, ya muy metido en vinos, vio como la historia de un rey de escarabajos gigantes - algo de escarabajo tenía, en efecto, el peto verde, escamado,

\footnotetext{
${ }^{6}$ Bakhtin, Rabelais And His World: 39-40. Refiriéndose a la función e importancia de la máscara Bakhtin comenta lo siguiente:

Even more important is the theme of the mask, the most important theme of folk culture. The mask connected with joy of change and reincarnation, with gay relativity and with the merry negation of uniformity and similarity; it rejects conformity to oneself. The mask is related to transition, metamorphoses, the violation of natural boundaries, to mockery and familiar nicknames. It contains the playful element of life; it is based on a peculiar interrelation of reality and image, characteristic of the most ancient rituals and spectacles. Of course it would be impossible to exhaust the intricate multiform symbolism of the mask. Let us point out that such manifestations as parodies, caricalures, grimaces, eccentric postures, and comic gestures are per se derived from mask. It reveals the essence of the grotesque.

${ }^{7}$ Podemos observar por medio de esta pregunta la falta de conocimiento que posee el curopeo sobre Amćrica, pues los incas fueron indios del Perú mientras que los azlecas son mexicanos.
} 
reluciente, del narrador-, que había vivido no hacía tanto tiempo, si se pensaba bien, entre volcanes y templos, lagos y teocallis, dueño de un imperio que le fuera arrebatado por un puñado de españoles osados, con la ayuda de una india enamorada del jefe de los invasores (p. 36).

Pero el mecanismo de la máscara no sólo sirve como mediador en la transformación del personaje en donde se anula su rol de amo, sino que el narrador adquiere la palabra (el enunciado histórico) de Montezuma, y, por tanto, mediante la máscara, hace suya la personalidad de Montezuma actualizando así su pasado indígena. El disfraz le permite camuflar las palabras del otro para hacer su propio enjuiciamiento del acontecer histórico de la conquista de México. Esto, por consiguiente, va creando un cambio significante en la novela, pues la historia de Montezuma y la conquista de México ya no es un hecho perteneciente al pasado histórico, sino que se actualiza y se convierte también en la propia historia del Amo en la búsqueda de su propia identidad.

Terminada la narración, el Amo, Montezumajuntoa los demás personajes, deciden irse a algún lugar tranquilo, fuera del bullicio del carnaval, donde les sea posible componer música. Van al "Ospedale della Pietă", y allí componen un gran "concerto grosso" combinando elementos étnicos americanos y europeos: "Ca-la-ba-son-son-son- cantaba Filomeno, ritmando cada vez más. Kabalasum- sum-sum- respondían el veneciano, el sajón y el napolitano" (p. 46). Mediante este canto afrocubano, se vuelven a unir los espacios, las realidades en la novela: América-Europa, para formar un sólo espacio textual en el suceder temporal de la música. Después del concierto deciden irse a descansar y van al cementerio. Al llegar al cementerio, el Amo-Montezuma empieza a sentir un gran cansancio y sueño: "Tengo sueño - decía Montezuma ... Tengo sueñorepetía el disfrazado" (p. 48). El sueño se convierte en otro de los mediadores en la transformación del personaje. El uso de la anáfora “tengo sueño" nos va revelando gradualmente esa transformación, primero aparece como Montezuma y luego como disfrazado. El participio "disfrazado" tiene un doble significante en el texto: disfraz y máscara encierran el doble aspecto del personaje, uno y otro, Amo/Montezuma, América/Europa, presente/pasado. Sacado de su sueño, el Amo es invitado de nuevo a narrar la "verdadera" historia de Montezuma:

El mexicano, sacado de su sopor, fue invitado a narrar nuevamente la historia de Montezuma que Antonio, la víspera, había mal ordo ensordecido como lo estaba por el griterio de las máscaras (p. 49).

El Amo adquiere en este momento el nombre genérico de "mexicano". El vocablo "mexicano" vendrá a limitar el espacio en contraposición con el vocablo "amo", de esta forma se intuye una sensación de autoridad, identificación y poder en la versión del Amo de la historia de México. Hay una aparente separación 
momentănea y un distanciamiento temporal, espacial, para poder narrar los hechos históricos acontecidos. Inmediatamente, ese espacio es reactivado, recuperado y actualizado en el presente cuando el mexicano, posesionado del momento histórico y de los personajes, narra la historia:

- iMagnífico para una ópera!" -exclamaba el pelirrojo, cada vez más atento al narrador que, llevado por el impulso verbal, dramatizaba el tono, gesticulaba, mudaba de voz en diálogos improvisados, acabando por posesionarse de los personajes (p. 49).

Es imprescindible hacer referencia al negro Filomeno y a su narración de la historia de su bisabuelo, Salvador, cuando emocionado por el hecho histórico, reencarna a los personajes de la historia y hasta los hace actuar:

$\mathrm{Y}$ alzando los brazos y accionando las manos como títeres, con los dedos pulgares y meñiques movidos como bracitos, continúa en la narración con tanta vida como la pone cualquier bululú de buen ingenio en sacarse personajes de trás de las espaldas y montarlos en el escenario de sus hombros. (-"Así cuentan algunos feriantes en los mercados de México - pensaba el viajero-la gran historia de Montezuma y Hornán Cortés") (p. 21).

Se observa aquí el paralelismo de ambas escenas: la narración de dos hechos distintos contados en la misma forma y actualizados con la misma emoción. Filomeno, orgulloso de su pasado histórico, deja de ser un mero narrador en tercera persona que narra los hechos objetivamente, para convertirse en protagonista/actor de la historia. Asi sucede con el Amo, quien deja de ser el narrador de la historia para posesionarse del discurso mismo y ubicar en un presente "su" pasado histórico de México. El narrador es el que realiza el discurso significante, se convierte en el sujeto de la enunciación y, por consiguiente, en el protagonista del enunciado ${ }^{8}$.

Una vez narrada la historia de Montezuma, el Fraile Antonio comienza a esbozar el argumento de lo que será la ópera de Montezuma. El Amo se despierta sobresaltado ante el deseo del Fraile de querer representar elefantes en la ópera: "En Mexico no hay elefantes - dijo el disfrazado, sacado de su

${ }^{8}$ La máscara es, en este momento, como dice Kristeva, la que "concentra, pues, la posibilidad del locutor de participar en su lenguaje como práctica significante de sí misma ...." Julia Kristeva, 232. Es, por tanto, la que aniquila al narrador y permite la transformación del narrador en actor o protagonista del hecho narrado.

${ }^{2}$ En 1936 Alejo Carpentier se entero, por medio de un músico italiano, Francisco Melipicro, de que otro músico, italiano también, Antonio Vivaldi, había compuesto una ópera de tema americano. Encuentra, más tarde la obra del poeta Alvise Guisti que proporcionó a Vivaldi el libreto de su "Montezuma" (1733). Esta le sirve de inspiración para escribir su obra Concierlo barroco. Es por ellos que el indiano conoce a Vivaldi, quien fascinado ante el disfraz de Montezuma, concibe esa misma noche la idea de escribir una ópera sobre la conquista de México, primera obra musical que se compuso en Europa sobre un tema americano. 
modorra por la enormidad del dislate" (p. 54). Vemos de nuevo que el Amo asume el papel de mexicano y se sitúa en el presente y en su experiencia de América para poder contar la historia, o refutar a aquellos que la interpretan erróneamente, según su propia convicción y modelos europeos. El AmoMontezuma se vuelve a dormir agobiado por el cansancio de la larga noche y al llegar a la pensión le despojan de su disfraz de cacique azteca. Con el dormir, y al despertar toma lugar un lapsus de tiempo en el que el "yo" histórico carece de existencia (ni tampoco de un inconsciente, puesto que no sueña). Sin embargo, al despertar se percibe una transformación de su identidad. Ya no es Montezuma, ni tampoco el Amo, ahora se convierte en el Indiano, aunque manteniendo en ese papel las otras dos personalidades. De esa forma, se va transformando paulatinamente de Montezuma a Indiano, identidad que le permite asumir una posición mucho más directa con el pueblo y la historia americana: "No era ya el Montezuma de la víspera ..."(p. 59).

Mientras ocurre esta transformación, el disfraz de Montezuma es utilizado para vestir a Massimiliano Miller, quien representará en la ópera el papel de Montezuma. Al quitarle el disfraz al Amo para vestir a Massimiliano Miller, se pasa al espacio escénico-ficticio de la historia de México: la ópera.

La ópera comienza con la representación de "la noche triste". Al empezar el primer acto, el Amo-indiano se da cuenta de que Teutile es una mujer y no el general de los ejércitos de Montezuma. De esta forma, lo representado para él no coincide con su conocimiento histórico. El Amo-indiano no acepta la interpretación histórica del Fraile Antonio, sobre la historia de México, ya que resulta ser, desde su punto de vista, una parodia y desfiguración de la historia, por eso grita:

- iFalso, falso, falso; todo falso! - grita. Y gritando "falso, falso, falso, todo falso, corre hacia el preste pelirrojo .... Ese final es una estupidez ... (p. 68).

El fraile pelirrojo le cita la crónica de Solís al indiano ${ }^{10}$, enfrentándonos de esta forma a otra de las características de la novela: la confrontación y generación de textos, es decir la intertextualidad ". La crónica de Antonio de Solís, Cronista mayor de Indias, hace penetrar la historia oficial de la Conquista en el texto ficticio. Esta se traspone del espacio histórico (la crónica) al espacio de la novela, como se demuestra en el siguiente cuadro:

Texto generador

1. Conquista de México
Autor

Antonio de Solís
1. La emperatriz, que sería de la misma edad, se hacía reparar el garbo y el espiritu con que mandaba el movimiemto y las ac-

\footnotetext{
- ${ }^{10}$ Antonio Solis, Historia de la conquisla de México (Chatillon-sur-seine: Imprenta de A. Pichat, s/f.)

${ }^{11}$ Sin embargo estos textos se des-mitifican y des-articulan unos a otros.
} 


\section{Texto generador}

1. Concierto Barroco
Alejo Carpentier ciones; pero su hermosura, más varonil que delicada ... (p. 553).

2. Dejo, algunos hijos: dos de los que le asistían en su prisión fueron muertos por los mejicanos cuando se retiró Cortés: y otros dos 6 tres hijas que se convirtieron después y se casaron con españoles ... (p. 373).

1. Y ahí se habla de una emperatriz, sí señor, mujer digna, animosa y valiente... Cap. XXV de la quinta parte (p. 68).

2. Y también se dice, en la cuarta parte, que dos o tres hijas de Montezuma se casaron con españoles (p. 68).

Carpentier trae a colación este pasaje donde se refiere a la emperatriz para refutar la idea de la veracidad histórica. Esta referencia crea en el lector una serie de preguntas; por ejemplo, ¿Tenía Guatimozín una emperatriz? ¿Sólo se concibe la historia de México desde el punto de vista europeo? ¿Tenía México emperatrices en esta época? ¿Se está imponiendo el modelo político europeo a la realidad de América? ${ }^{12}$.

La ópera sirve en la obra como otro elemento mediador, pues el indiano, ante la representación que acaba de ver, se siente ofendido, molesto, reconoce su linaje español, y sobre todo por su re-acción ante la parodia histórica, asume la verdadera personalidad e identidad de indiano, americano y español: se encuentra a sí mismo, se centraliza, se ubica en su historia. Por lo tanto los términos que en un principio estaban en oposición, descentralizados en la división del ser en la novela: americano/europeo, pasado/presente, acá/allá, en

\footnotetext{
${ }^{12}$ La mención de las hijas de Montezuma y su casamiento con españoles también parece venir a colación, como una posible parodia y transformación histórica. Observamos, mediante esta confrontación de texto, que ambos dialogan entre sí, y los dos términos opuestos, americano/europen, son llevados al extremo de quedar al fin reconciliados en una ambigüedad. Estos hechos aparecen fragmentados, tomando del acontecer histórico los aspectos positivos de la conquista y eliminando los negativos:

...-" ¿Y ese Teutile que se nos vuelve sombra?" - "Tiene un nombre pronunciable, que puede darse a una mujer". - "YYqué se hizo de Guatimozín, el héroe verdadero de todo esto?" - "Hubiera roto la unidad de la acción. Sería personaje para otro drama". - Pero ... Montezuma fue lapidado." — Muy feo para un final de ópera ... Aquí la gente viene al teatro a divertirse". - ¿Y dónde meticron a Doña Marina, en toda esta mojiganga mexicana? - La Malinche esa fue una cabrona traidora y el público no gusta de traidoras. 69 .
} 
el transcurso de la acción por la ambivalencia y la transformación (el viaje, el carnaval, la máscara, la ópera) terminan centralizados y unidos en el "yo" espectador e histórico del Amo-Indiano-Mexicano. El yo "esquizofrénico" Amo-Montezuma-Indiano-ahora quedará unido en uno: americano. Dado que la ópera concretiza su "encuentro" con su identidad, el proceso transformacional del Amo que se dio en su re-acción a los espacios visitados - Cuba, España e Italia-lo sitúa en el espacio original -México. En la ópera culmina el proceso transformacional del Amo con la recuperación y afirmación tanto de su propia y verdadera patria como de su verdadera historicidad americana. 